\title{
A Non-conventional Approach to Understanding the Geographical Influence on the Transmission of SARS-CoV-2 and IFR
}

\author{
UdAYA KETIPEARACHCHI ${ }^{\star 1}$ \\ ${ }^{1}$ Albany Road, Coventry, CV5 6NF, UK \\ *Corresponding author: uketipearachchi@yahoo.co.uk
}

Compiled April 30, 2021

\begin{abstract}
Transmission of SARS-CoV-2 is not well understood and different models are needed (i.e. one model cannot answer it all) to understand and fight this disease. Here, we present two models based on indirect transmission of SARS-CoV-2 that explain the influence of ambient temperature and air pollution on outdoor and indoor behavior of SARS-CoV-2. These models discuss the temperature dependency of the lethality of SARS-CoV-2 and its spread during indoor and outdoor exposure. Furthermore, the temperature effect on the half-life of SARS-CoV-2 is discussed if the SARSCoV-2 is transmitted via particulate matter or surfaces. It is also important to understand the role of dew point instead of the humidity factor alone as the combined effect of temperature and humidity might play a major role in the transmission of SARS-CoV-2 .

Key Words : Half-life ; Strength of viral dose ; Temperature effect ; Dew point. ๑ 2021 Optical Society of America

http://dx.doi.org/10.1364/ao.XX.XXXXXX
\end{abstract}

\section{INTRODUCTION}

Although the SARS-CoV-2 outbreak in Wuhan, China was reported initially to the World Health Organization(WHO)in December 2019, there is no clear understanding of its spread and survival up until now and therefore, there is genuine urgency in proposing different models ${ }^{1,2}$. This uncertainty has a huge impact on our health, economy, and politics, and therefore it is important to eradicate this uncertainty sooner than later. However, according to Worldometer SARS-CoV-2 related data, it seems like there is a geographic (human and physical) factor involved in the IFR (Infection Fatality Ratio). This is evident by the low IFR for highly populated countries (e.g. Singapore, Bahrain, etc.) and the high IFR for many European countries. For example, IFR in Singapore (population density is 7810 people per square kilometer respectively) is 0.049 whereas in the UK ( population density in London and the UK are 5630 and 275 people per square kilometer respectively) it is 2.79 as of $4^{t}$ ${ }^{h}$ January 2021 (Worldometer data). To understand the transmission and the variations in IFR, there are factors that have to be taken into account such as UVB intensity of sunlight, dew point, temperature, pollution (e.g.PM2.5 particle density), indoor ventilation, population density, social events,immunity, age, lifestyle, underlying health conditions, unknown genetic factors, etc. As per the WHO scientific brief circulated on 29 March 2020, SARS-CoV-2 is primarily transmitted between people through respiratory droplets and contact route. ${ }^{3-5}$ Transmission via respiratory droplets can be in the form of sneezing,coughing, or speaking. Tiny respiratory droplets filled with pathogens can transmit infection when they move directly from the respiratory system of an infectious individual to a susceptible mucosal membrane of a recipient, generally within a short distance. In general, large droplets $(>5 \mu)$ fall rapidly to the ground under gravity or nearby surfaces whereas the small particles $(<5 \mu)$ can remain suspended in the air for a significant period. These small droplets can enter someone's respiratory system when two people are in close proximity $(<1 \mathrm{~m})$. Some studies have revealed that environmental surfaces play an important role in the endemic and epidemic transmission of certain pathogens that cause infections. ${ }^{6}$ Although many respiratory viruses are believed to transmit over multiple routes, of which droplet and aerosol transmitted paths are involved, their significance in transmitting the disease is unclear. ${ }^{7}$ It was also reported that WHO guidelines such as hand washing and maintaining social distance do not prevent infection by small droplets exhaled by an infected person that can travel distance of meters or tenth of meters in the air and carry their viral content. ${ }^{8}$ According to literature, the public health authorities have marginalized the significance of airborne transmission of SARS-CoV-2 or influenza could be due to the problems associated with the detection of the viruses in the air. ${ }^{8}$ Having said that it was reported that aerosol and fomite transmission of SARS-CoV-2 is plausible, since the virus can remain viable and infectious in aerosols for hours and on surfaces up to days. ${ }^{9}$ Therefore, it is important to understand any involvement of air pollution in the transmission of viruses. PM2.5, a pollutant refers to atmospheric particulate matter that has a diameter of fewer than 2.5 microns, which may have the potential for transmitting SARS-CoV-2. Respiratory droplets can contaminate this PM2.5 and dehydrate over time as water content (99\%) in saliva can evaporates during dehydration. ${ }^{10,1} 1$ As a result, SARS-CoV-2 can adhere to the surface of PM2.5 depending on the electrostatic attraction, 
contact angle and bonding capability between the PM2.5 and the SARS-CoV-2. Eventually, it may easily enter our respiratory system while leading to an outdoor spread. The lethality of the dose depends on the amount of virus bound to particulate matter. According to global health observatory (GHO) data, the mean ambient air pollution of particulate matter with an aerodynamic diameter of $2.5 \mu \mathrm{m}$ ranges from less than 10 to over $100 \mu \mathrm{g} / \mathrm{m}^{3}$ in urban areas. This implies that around 769000 PM2.5 particles are suspended in a cubic meter of air if the PM2.5 level is $10 \mu \mathrm{g} / \mathrm{m}^{3}$ and this is enough to spread the disease. These particles are released from a variety of indoor and outdoor activities. For example, burning candles, cooking, forming complex reactions of gaseous pollutants emitted by household cleaning products and air fresheners, smoking, etc. contribute to indoor pollution whereas emission from vehicles, industries, power plants, etc.contribute to outdoor pollution. However, being exposed to a lethal dose of SARS-CoV-2 will develop a more severe illness, and therefore, it is worthwhile to understand the strength of SARS-CoV-2 dose (i.e. harmfulness) and its transmission.

\section{SURVIVAL OF COVID-19}

it was revealed that simulated sunlight rapidly inactivates SARSCoV-2 on surfaces. ${ }^{12}$ Anyway, saliva can act as a shield for protecting SARS-CoV-2 from exposure to UVB (280-315nm) as saliva absorb good portion of $\operatorname{UV}(\lambda>220 \mathrm{~nm})$ light. Therefore, it is important to remove this shield to inactivate SARS-CoV-2, and evaporation of saliva depends on the dew point and the ambient temperature. Although there have been some studies on the temperature and relative humidity effect on SARS-CoV-2 trans- mission, different models are needed for in-depth analysis of SARS-CoV-2 transmission and its effects. ${ }^{13,14}$ It was also reported that the growth rate of some viruses and bacteria declines slowly with decreasing temperature and more rapidly upon temperature increase. ${ }^{15}$

\section{APPROACH}

We believe that in-depth studies on the behavior (strength of the viral dose and its survival in our environment) of SARSCoV-2 in the ambient temperature, before entering our body, will help us understand its spread and lethality. For example, a study revealed that increasing the temperature while maintaining humidity drastically reduces the survivability of the SARS-CoV-2 (i.e. temperature affects the half-life of SARS-CoV2). ${ }^{16}$.Therefore, this investigation proposes two mathematical models based on two different hypotheses to understand the effects of temperature and air pollution on the strength of SARSCoV-2 dose. However, the term "strength of SARS-CoV-2 dose" used in this non-conventional approach is different from the term "infectious dose" which is the amount of virus necessary to make a person sick or create an infection. Nevertheless, the strength of SARS-CoV-2 dose refers to the chemical potential of SARS-CoV-2 $(\mu)$ at a certain temperature and number of active SARS-CoV-2 (N) in that dose at that temperature (i.e. temperature may have an effect on the chemical potential of individual virus and half-life of a virus). However, the chemical potential of SARS-CoV-2 vary after entering the human body whereas number of active SARS-CoV-2 virus remain same prior to its internalization. Eventually, proteolytic enzymes (RNA olymerase) are involved in the replication of SARS-CoV-2 in which the factor "Strength of SARS-CoV-2 dose" will play an important role. In this non-conventional approach, the strength of SARS-CoV-2
dose(A) before entering human body is expressed as a function of temperature, $\mathrm{f}(\mathrm{t})$ as follows.

$$
A=\kappa \mu N=\kappa f(t)
$$

where $\mathrm{k}$ is considered as a constant, even though it may depend on other physical parameters (e.g. dew point, etc). Furthermore, this temperature effect on the strength of SARS-CoV-2 dose is discussed under two different ways of transmission of SARSCoV-2 such as direct and indirect transmission of SARS-CoV-2

\section{HYPOTHESIS 1}

SARS-CoV-2 is dormant at very low temperatures and its activity increases with increasing temperature and then decreases. Here is one of the evidences we have to date to support this hypothesis. It was reported that human metapneumo virus (HMPV) is most prevalent when the temperature becomes slightly warmer $(7.4 \mathrm{C}) .{ }^{17}$. In this case, we assume that SARS-CoV-2 is transmitted via dust particles (e.g. PM2.5, aerosols, etc.) and shows its highest strength at temperature $\mathrm{T}(\mathrm{K})$. In general, virus droplets remain infectious for a time depending on where they fall (e.g. surface or dust particle). The interaction between SARS-CoV-2 and dust particles (e.g.PM2.5) depends on surface properties of the particle. However, the survival time of the SARS-CoV-2 depends on the surface type and ambient temperature and it was estimated that the median half-life of SARS-COV-2 was approximately 5.6 hours on stainless steel and 6.8 hours on plastic. ${ }^{9}$ Now rearrange the equation (1) to fit this situation as follows

$$
A=\alpha D\left[t-0.5(t-T+1)^{2}\right]
$$

where D is SARS-CoV-2 contaminated particle density in air and it is assumed that all the particles equally contaminated with the virus, $t$ is the ambient temperature, $\alpha$ is a dew point dependant. Therefore, the virus shows its highest strength when the temperature $(t)$ reaches $T(K)$. To construct a graph, let us give some random values to the constants in the above equation such that $\alpha=0.4, \mathrm{~T}=275 \mathrm{k}$, and $\mathrm{D}=0.14$. Now let's look at the graph in Fig. 2 which shows its highest strength 15.372 a.u. at $275 \mathrm{~K}$.

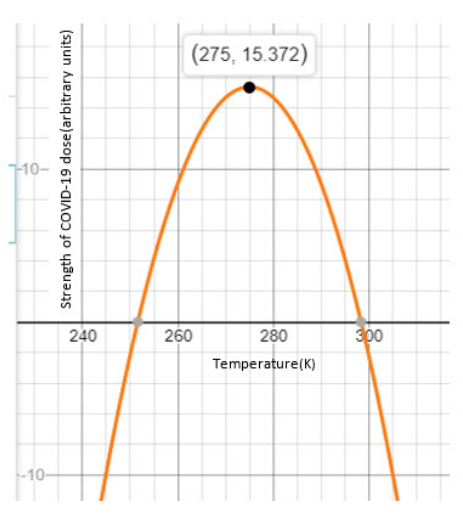

Fig. 1. Graphical illustration of temperature effect on strength of SARS-CoV-2 for indirect transmission of SARS-CoV-2 according to hypothesis-1. 


\section{HYPOTHESIS 2}

SARS-CoV-2 is not dormant at low temperatures and become weak at high temperatures

Here, we consider a SARS-CoV-2 transmission via dust particles (PM2.5) and expect that the strength of SARS-CoV-2 dose is constant at low temperature and starts to decline over time (i.e. half-life is temperature-independent at low temperatures). For example, it was reported that in a guinea pig model, IAV transmission is more effective in cold and dry conditions ${ }^{18}$. This phenomenon is explained mathematically in equation (5).

$$
A=\beta D\left[\Lambda-e^{\varphi(t-T)}\right]
$$

Where $t$ is the ambient temperature, $\mathrm{T}$ is the temperature at which the activity of virus is stable, D is the virus density of the contaminated surface and it is assumed that all the particles are equally contaminated, $\beta$ is a dew point dependent, $\Lambda$ and $\varphi$ are constants. Let's give some arbitrary values to the constants in the equation (3) as we did previously. Suppose $D=2, \beta=2.5, \Lambda$ $=1, \mathrm{~T}=333 \mathrm{k}$ and $\varphi=0.1$. Then, the graph in Fig. 4 explains that the strength ofSARS-CoV-2 is going to decline after $280 \mathrm{~K}$ and dies at $333 \mathrm{~K}$.

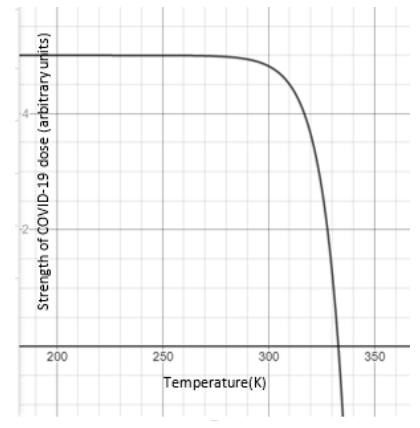

Fig. 2. Graphical illustration of temperature effect on strength of SARS-CoV-2 for indirect transmission of SARS-CoV-2 according to hypothesis-2

\section{DISCUSSION}

However, temperature alone cannot account for the global variation in IFR, but according to this model, temperature factor plays an important role. The constants $\alpha$ and $\beta$ are elements of $\kappa$ in the equation (1) (i.e. $\kappa=\{\alpha, \beta\}$ ) and Fig.1 and Fig. 2 are drawn for illustration purposes only. The spread of a virus depends on its ability to survive in the environment, and the lethality of the viral dose is judged by the strength of the SARS-CoV-2 dose assuming that the direct transmission is suppressed as a result of following WHO guidelines. Anyway, in the case of indoors, exposure to a small dose of SARS-CoV-2 for prolonged period, especially with poor ventilation, can contribute to the IFR. For example, ventilation is poor during winter in most of the enclosed spaces and the SARS-CoV-2 contaminated fomites (e.g. PM2.5, aerosols) can carry a small dose but exposing someone to SARS-CoV-2 contaminated air for a prolonged period is dangerous. On a different note, the heavy droplets deposited on surfaces can float in the air if the adhesion between the surface and SARS-CoV-2 is poor (i.e. SARS-CoV-2 can float in the air after water in the saliva is evaporated). The magnitude of SARS-CoV-2 contamination also depends on the size of the enclosed space and number of infected people (i.e. asymptomatic carriers) live within this enclosure. The outdoor spread of SARS-CoV-2 depends on many factors such as dew point, temperature, UVB intensity, etc. Although the outdoor transmission of SARS-CoV-2 may not significantly affect IFR if the social distancing is maintained and vulnerable people are confined to indoors (i.e. a small virus dose from air/contaminated surfaces may have an ability to make an infection for vulnerable people), indoor exposure to SARS-CoV-2 may contribute to IFR. In contrast, weather pattern and surface properties of particulate matter may contribute to the outdoor spread of SARS-CoV-2 whereas building structure(i.e. ventilation, surface properties of interior surfaces, etc.), relative humidity, the freshness of the air, immunity of the people live in enclosed spaces and their underline health conditions, number of infected people live within that enclosure, etc. may contribute to IFR.

\section{CONCLUSIONS}

In general, both direct and indirect transmission of SARS-CoV-2 contributes to the IFR and spread. It is also reported that the temperature has an important effect on viral activity, particularly in the case of enveloped viruses like SARS-CoV-2. ${ }^{17}$ Therefore, two different mathematical models are discussed in this communication to understand the temperature effect on IFR . According these modals, indirect transmission could have a temperature effect on "strength of SARS-CoV-2" and its transmission assuming that direct transmission has no considerable temperature effect (i.e. SARS-CoV-2 enter the human body within seconds). Respiratory droplets contaminate particulate matter or surfaces and suspend in the air to assist the spread after the dehydration. The strength of the SARS-CoV-2 dose is a measure of the lethality of the viral dose which may be used to understand the variation in the IFR. Ambient temperature may affect half-life of SARS-CoV-2, which influences the lethality of the viral dose. Although temperature reduces the lethality of SARS-CoV-2 dose, the transmission of SARS-CoV-2 is not significantly controlled by the temperature as a small amount of virus is enough for the transmission. In contrast, the proposed models might be used to benchmark the lethality of SARS-CoV-2, and a better understanding of the transmission of SARS-CoV-2 and its lethality will help control the SARS-CoV-2 impact on our lives. On a different note, the geographical influence on transmission of SARS-CoV-2 and IFR may change over time due to the adaptions of SARS-CoV-2 to different environments and formation of new variants.

\section{REFERENCES}

1. Panovska-Graffiths J, Can mathematical modelling solve the current Covid-19 Crisis?. BMC Public Health, 2020;20:551.

2. Holmdahl I. S. M, et al. Wrong but Useful-What Covid-19 Epidemiologic Models Can and Cannot Tell Us. N Engl J Med 2020, at NEJM.org. DOI: 10.1056/NEJMp2016822.

3.Liu J. et akl. Community transmission of severe acute respiratory syndrome coronavirus 2. Shenzhen, China, 2020. Emerg Infect Dis 2020 doi.org/10.3201/eid2606.20039.

4.Hung c. et al. Clinical features of patients infected with 2019 novel coronavirus in Wuhan, China.Lancet,2020;395:497-506.

5.World Health Organization Report of the WHO-China Joint Mission on coronavirus Disease 2019(COVID-19) 16-24 February 2020[internet]. Geneva: World Health 
Organization, 2020. (https://www.who.int/docs/defaultsource/coronaviruse/who-china-joint-mission-on-covid-19final-report.pdf.)

6.Otter J. A. et al. Evidence that contaminated surfaces contribute to the transmission of hospital pathogens and an overview of strategies to address contaminated surfaces in hospital settings. American Journal of infectious Control 2013;S6:511.

7.Jayaweera M. et al. Transmission of COVID-19 virus by droplets and aerosoles: A critical review on the unresolved dichotomy. Environmental Research 2020;188:109819.

8. Morawska L. et al. Airborne transmission of SARS-CoV-2: The world should face the reality. Environment International 2020;139:105730.

9. Doremalen N. V. et al. Aerosol and Surface Stability of SARSCoV-2 as Compared with SARS-CoV-1. N Engl J Med 2020;382;16.

10.Stadnytskyi V. et al. The airborne lifetime of small speech droplets and their potential importance in SARS-CoV-2 transmission. PNAS 2020;117:11877.

11. Humphrey S. P. et al. A review of saliva: Normal composition, flow, and function.The Journal of Prosthetic Dentistry 2001;85:162-169.

12.Ratnesar-Shumate S. et al. Simulated Sunlight Rapidly Inactivate SARS-CoV-2 on surfaces. Infectious Diseases Society of America 2020;222:214-222.

13.Casanova L. M. et al. Effects of Air Temperature and Relative Humidity on Coronavirus Survival on Surfaces.Appl. Environ. Microbiol. 2010;76:2712-2717.

14.Chan K. H. et al. The Effects of Temperature and Relative Humidity on the Viability of the SARS Coronavirus.Advances in Virology 2011;doi:10.1155/2011/734690.

15.Cooper V. S. et al. Evolution thermal dependence of growth rate of Escherichia coli population during 20,000 generation in a constant environment. Evolution 2001;55:889-896.

16. Riddell S. et al. The effect of temperature on persistence of SARS-CoV-2 on common surfaces. Virology Journal. 2020;17,145.

17.Prince R.H. et al. Association between viral seasonality and meteorological factors. Scientific reports.2019;9.

18. Lowen, A. C.et al. Influenza virus transmission is dependent on relative humidity and temperature.PLoS Pathog 2007; 3, 1470-1476, https://doi.org/10.1371/journal.ppat.0030151

(2007). 